\title{
Protection générale des ressources en eau et périmètres de protection des captages d'alimentation en eau potable
}

\author{
Global protection of water resources and protection areas \\ of drinking water catchment areas
}

par G. Alcaydé

Conseil Supérieur d'Hygiène Publique de France

Since the natural protection of underground water against physical, chemical and microbiological pollution varies considerably depending on the geological and hydrogeological context, extra protective measures have been put in place by regulations.

In France, the provisions of general regulations relating to the protection of resources have been reinforced by specific regulations aiming to reduce the risks of pollution around drinking water catchment areas. These regulations provide for the setting up of protection areas around the catchment areas ; a proposed ranking of the protection areas is given according to the risks, and a possible approach to their definition is set out.

\section{I —INTRODUCTION}

La France est riche en eaux souterraines puisqu'elle possède environ deux cents aquiferes d'importance régionale (dont vingt-cinq aquifères captifs) qui fournissent environ $60 \%$ des eaux de consommation pouvant souvent être distribuées sans traitement.

Ces eaux sont « cachées " et, en dépit des apparences, elles sont souvent fragiles et les pollutions dont elles sont l'objet sont d'autant plus graves que, contrairement à ce que l'on observe pour les eaux de surface, elles ne peuvent se résorber que très lentement, même lorsque la source polluante est tarie. Il est donc évident que, dans ce domaine, il vaut mieux prévenir que guérir car la dépollution coûte beaucoup plus cher que la prévention.

Si l'on observe globalement l'évolution de la qualité des eaux souterraines, on constate leur dégradation lente mais croissante avec notamment une augmentation régulière des teneurs en nitrates, en pesticides et l'apparition de pollutions chimiques et microbiologiques qui sont susceptibles d'avoir des effets néfastes sur la santé lorsqu'elles affectent les eaux d'alimentation.

Cette altération de la qualité est possible parce que la plupart des eaux souterraines ne sont pas totalement protégées naturellement contre les pollutions et il a donc été nécessaire de prendre des mesures réglementaires de prévention à différents niveaux pour sauvegarder la qualité des ressources en général et, avec des objectifs différents, de celles captées à des fins alimentaires. Encore convient-il d'appliquer correctement et à bon escient des réglementations généralement bien conçues, ce qui n'est pas toujours le cas.

\section{II — RESSOURCES EN EAU SOUTER- RAINE : CARACTÉRISTIQUES ET PROTECTION NATURELLE}

\footnotetext{
2.1 Principales catégories de réservoirs d'eau souterraine
}

On peut en distinguer trois :

\subsubsection{Les réservoirs homogènes}

Ils sont généralement constitués par des sables et des graviers. Ils présentent une perméabilité d'interstices ouverte c'est-à-dire que l'ensemble des vides de la roche est formé par les espaces intergranulaires communiquant entre eux. 
Exemples : alluvions, formations sableuses des bassins sédimentaires, etc.

\subsubsection{Les réservoirs hétérogènes}

Ils sont constitués principalement par des calcaires, des roches granitiques, des roches volcaniques, etc. Il présentent une perméabilité de fissures. Lorsque dans les calcaires les fissures sont ouvertes, on parle de réservoir karstique, l'eau pouvant alors circuler à des vitesses élevées dans les fissures.

\subsubsection{Les réservoirs mixtes}

Dans ceux-ci coexistent perméabilités d'interstices et de fissures.

Exemples : craie, grès, etc.

\subsection{Différents types de nappes}

On distingue :

\subsubsection{Les nappes libres}

Celles-ci, à partir d'un substratum imperméable, occupent un aquifère sur une certaine hauteur sans rencontrer d'obstacle (écran imperméable) limitant leur développement vers le haut. Elles sont en général peu profondes.

\subsubsection{Les nappes captives}

Généralement profondes à semi-profondes, elles sont contenues dans un terrain poreux interstratifié entre deux horizons imperméables formant respectivement son mur et son toit. Dans ce cas, l'aquifêre est saturé sur toute sa hauteur.

\subsection{Ecoulement des eaux souterraines}

Les eaux souterraines ne stagnent pas. Elles sont en permanence en mouvement et, en conditions naturelles, un aquifère est en état d'équilibre dynamique.

Lorsque l'on pompe dans un aquifère, on rompt l'état d'équilibre dynamique et l'on provoque un rabattement plus ou moins important de la surface de la nappe autour du point de prélèvement, ce qui a pour effet de modifier les conditions d'écoulement de l'eau (sens, vitesse, etc.).

\subsection{Protection naturelle des eaux souterraines}

Elle dépend essentiellement des éléments suivants :

\subsubsection{Du type de nappe}

Si les nappes captives sont naturellement bien protégées, les nappes libres présentent des vulnérabilités variables.

\subsubsection{De la nature des formations de recouvrement}

- Une couche argileuse continue assure naturellement une protection efficace en limitant l'infiltration d'eaux polluées, en fixant certains contaminants, en favorisant la dégradation de certaines substances polluantes (oxydation, action des microorganismes, etc.),

- une couverture sableuse assurera une bonne filtration et permettra d'éliminer rapidement les matières en suspension et les microorganismes mais sera sans efficacité vis-à-vis d'autres polluants, qu'ils soient ou non solubles dans l'eau (sels, pesticides, hydrocarbures, solvants, etc.).

\subsubsection{De l'épaisseur du recouvrement}

Lorsque celle-ci est importante, les eaux de percolation et les polluants éventuels mettent plus de temps pour atteindre la nappe souterraine, ce qui favorise la dégradation ou le piégeage de certaines substances polluantes et réduit d'autant le risque de contamination de l'aquifere.

\subsubsection{De la vitesse d'écoulement des eaux dans la nappe}

Plus celle-ci est lente, plus les phénomènes de dilution, de dégradation, de fixation des produits polluants joueront. Ainsi, en nappe d'insterstices, cette vitesse peut être très lente alors qu'elle est élevée en milieu fissuré : le transfert d'un égal volume d'eau peut nécessiter un an dans les alluvions et de un quart d'heure à 20 jours en milieu karstique.

\subsubsection{Du pouvoir filtrant de la roche réservoir}

Dans le cas d'un aquifère sableux, l'épuration microbiologique est rapide alors qu'en milieu fissuré, l'eau circule dans des terrains dépourvus de pouvoir filtrant, ce qui permet un transfert rapide et lointain des matières en suspension, des polluants et des microorganismes.

Etant donné que, dans de nombreux cas, les réservoirs aquiferes souterrains ne possèdent pas une protection naturelle suffisante, et que le développement des activités engendre de nombreuses pollutions, tant diffuses que ponctuelles, les pouvoirs publics ont été conduits à prendre, par voie législative et/ou réglementaire, un certain nombre de mesures afin d'assurer une meilleure préservation des ressources en général et de renforcer la protection autour des points de prélèvement d'eau destinée à l'alimentation.

\section{III —DISPOSITIONS LÉGISLATIVES ET RÉGLEMENTAIRES POUR LA SAU- VEGARDE DES EAUX SOUTER- RAINES}

Outre les lois sur l'eau du 16 décembre 1964, du 3 janvier 1992 et la loi du 19 juillet 1976 relative aux installations classées pour la protection de l'environnement, de nombreux textes ont défini les dispositions à prendre pour préserver la qualité des eaux souterraines. On ne peut en dresser ici une liste exhaustive mais on peut rappeler, à titre d'exemples, qu'ils fixent notamment :

- les mesures nécessaires à la restauration et à la préservation de la qualité des eaux,

- les conditions dans lesquelles peuvent être interdits ou réglementés :

- les déversements, écoulements, jets, dépôts directs ou indirects d'eau ou de matières susceptibles d'altérer la qualité des eaux,

- la mise en vente et la diffusion des produits ou dispositifs susceptibles de nuire à la qualité des eaux,

- les forages, prises d'eau, travaux ou ouvrages de rejet,

- les dispositions à respecter par les installations classées pour la protection de l'environnement,

- les programmes d'action à mettre en œuvre dans les zones vulnérables en vue de la protection des eaux contre la pollution par les nitrates d'origine agricole,

- l'agrandissement ou la création de cimetières,

- les stockages souterrains de gaz. 


\section{IV —DISPOSITIONS LÉGISLATIVES ET RÉGLEMENTAIRES SPÉCIFIQUES À LA PROTECTION DES CAPTAGES D'EAU DESTINÉE À LA CONSOM- MATION HUMAINE}

La réglementation générale relative à la sauvegarde de la qualité des eaux souterraines a été complétée par des textes spécifiques permettant de renforcer les mesures de protection autour des captages destinés à l'alimentation humaine. Ceuxci n'ont pas pour objet de renforcer la protection de la nappe exploitée - ce qui est fait par la réglementation générale mais a pour mission essentielle de sauvegarder la qualité de l'eau dans une zone rendue sensible par le prélèvement qui est opéré.

L'article L.20 du Code de la santé publique et ses textes d'application ont institué trois périmètres de protection autour des points de prélèvement d'eau, les deux premiers cités étant obligatoires :

- un périmètre de protection immédiate englobant le ou les points de prélèvement.

- un périmètre de protection rapprochée qui doit protéger efficacement le captage vis-à-vis de la migration des substances polluantes.

- un périmètre de protection éloignée qui, si nécessaire, prolonge le précédent pour renforcer la protection contre les pollutions.

Il s'agit, en fait, « d'une protection complémentaire dont l'objectif est de préserver les points de prélèvement des risques de pollution provenant des activités exercées à proximité " (Circulaire du 24 juillet 1990 - J.O. du 13 septembre).

\section{V שUTILITÉ ET FINALITÉS DES PÉRIMÈTRES DE PROTECTION}

La mise en place des périmètres de protection nécessite une procédure généralement lourde, longue à mettre en œuvre et qui peut se révéler coûteuse pour la collectivité si celle-ci se trouve dans l'obligation d'indemniser les propriétaires touchés par la création de servitudes.

Bien que l'utilité d'une telle protection soit admise par la grande majorité elle est néanmoins, pour les raisons qui viennent d'être évoquées, contestée par certains qui considèrent qu'il est possible d'abandonner cette obligation, la réglementation générale pouvant, à leurs yeux, être suffisante pour assurer la protection des ressources en eau.

La question est donc posée de la réelle utilité des périmètres de protection et, pour tenter d'y répondre, il convient de s'interroger sur leur hiérarchisation et sur leurs finalités.

\section{- 5.1 Hiérarchisation des périmètres de protection}

Les périmètres de protection peuvent être hiérarchisés en fonction de trois types de risques:

1 - le risque d'introduction directe de polluants dans l'ouvrage de prélèvement qui peut être prévenu par la création du périmètre de protection immédiate.

2 - le risque de dépassement d'au moins une des limites de qualité réglementaire de l'eau à la suite d'une pollution qui peut être maitrisé par la mise en place du périmètre de protection rapprochée.

3 - le risque de dégradation de la qualité de l'eau sans que celle-ci soit pour autant rendue impropre à la consommation qui peut être maîtrisé en créant un périmètre de pro- tection éloignée comprenant la zone où un tel effet pourrait se faire sentir.

Seraient donc « hors périmètres de protection " toutes les autres zones où une pollution ne pourrait dégrader la qualité de l'eau captée et où ne s'appliquerait que la réglementation générale relative à la protection des eaux.

Il apparaît donc nettement que les rôles dévolus aux périmètres de protection sont définis, sans ambiguïté, par rapport à des objectifs de protection de la santé publique et que les dispositions réglementaires correspondantes sont justifiées et parfaitement cohérentes.

\subsection{Objectif à atteindre : protection absolue ou limita- tion des risques?}

En cas d'épandage ou de déversement de contaminants, plusieurs cas peuvent se présenter:

$1^{\text {er }}$ cas - Le déversement a lieu à l'extérieur de la zone d'alimentation du captage :

- il n'y a aucun risque de pollution de l'eau captée.

$2^{e}$ cas - Le déversement a lieu dans la zone d'alimentation : la concentration pourra être, suivant la nature du polluant et la quantité déversée :

- nulle,

- proche de la concentration d'émission.

- avoir toutes les valeurs possibles entre ces extrêmes.

Le problème posé est donc complexe et quels que soient les moyens mis en œuvre, aucun découpage de la zone d'alimentation d'un captage (périmètres de protection) ne permettra de garantir de façon parfaite la protection de celui-ci.

Il est donc évident qu'on ne peut espérer une protection absolue des ouvrages de captage et que l'on doit donc viser la limitation des risques.

\subsection{Démarches possibles pour une limitation des risques}

On peut considérer qu'il existe quatre possibilités :

\subsection{Une démarche « minimaliste »}

Celle-ci est prônée par ceux qui estiment que la réglementation générale est suffisante pour assurer la protection des captages.

Outre le fait que l'efficacité d'une telle démarche est douteuse, il serait indispensable de renforcer la réglementation générale et de l'appliquer de façon rigoureuse à l'ensemble du territoire, ce qui ne serait pas sans effets négatifs, sur le plan économique notamment, car l'on serait amené à créer des contraintes aboutissant à une limitation des activités dans des secteurs où cela ne s'imposerait pas.

\subsubsection{Une démarche " maximaliste "}

Elle consiste à assurer la protection de la totalité de la zone d'alimentation du captage en y proscrivant toutes les causes de pollution.

Si cette démarche est défendable sur le plan hydrogéologique, on ne peut raisonnablement envisager de l'appliquer partout en raison des superficies concernées qui peuvent être très grandes et de la disproportion entre la sévérité des contraintes et les avantages d'une sécurité maximum.

Elle peut être acceptable dans deux cas :

- celui de bassins d'alimentation d'étendue limitée dans lesquels la vulnérabilité des eaux serait forte,

- celui des captages d'eaux minérales. 


\subsubsection{Une démarche " traditionnelle "}

Elle vise, dans l'esprit des textes réglementaires, à délimiter des périmètres de protection dans lesquels sont définies des contraintes et des mesures préventives adaptées pour limiter les risques les plus graves, les plus apparents ou les plus prévisibles.

Il s'agit alors, pour l'hydrogéologue, de faire ses propositions sur la base d'un diagnostic et d'un pronostic fondés sur l'interprétation d'observations et d'informations le plus souvent qualitatives. Dans ce cas sont pris en considération la protection naturelle de la nappe sollicitée, les caractéristiques de l'aquifère, la piézométrie, la vitesse et le sens d'écoulement de l'eau, etc.

Si cette démarche présente des inconvénients et des imperfections, elle a l'avantage d'être adaptable à la variété des cas possibles et peut même être couplée avec la démarche "dynamique " lorsque celle-ci peut être mise en œuvre.

\subsubsection{Une démarche "dynamique »}

Elle consiste à déterminer dans la zone d'alimentation du captage des courbes isochrones représentatives du temps de transfert d'un polluant, déversé en un point quelconque, jusqu'au captage. On définit alors les limites des périmètres de protection en fonction du délai jugé nécessaire pour intervenir avant que l'eau captée soit polluée.

Séduisante dans son principe, cette démarche a ses limites car elle suppose :

- que l'on sache déterminer correctement les isochrones, ce qui n'est possible, en pratique, que par couplage d'un modèle d'écoulement et d'un modèle de dispersion. La qualité des résultats dépend évidemment de la qualité du modèle, donc des valeurs retenues pour les paramètres hydrauliques.

- que le déversement du polluant soit détecté le plus tôt possible et en tout cas avant l'arrivée du front de pollution au droit de l'isochrone choisie, ce qui suppose la mise en place de dispositifs d'alerte fiables (piézomètres de suivi).

De plus, il faut signaler qu'en l'état actuel des techniques, toute modélisation fiable dans les aquifères fissurés est exclue.

\section{VI $\square$ CONCLUSION}

L'adoption par voie législative et réglementaire de mesures visant à la sauvegarde de la qualité des ressources en eau trouve sa justification dans le fait que la protection naturelle des nappes d'eau souterraine n'est pas toujours suffisante pour assurer efficacement leur protection contre les pollutions, qu'elles soient diffuses ou ponctuelles.

Le dispositif réglementaire mis en place pour la protection générale des ressources a été complété par des mesures spécifiques, avec création de périmètres de protection autour des points de prélèvement d'eau destinée à l'alimentation humaine. Dans ces derniers il est possible de renforcer notablement les mesures de protection ce qui permet d'éviter de créer inutilement de lourdes contraintes sur l'ensemble du territoire national. Ces périmètres de protection sont définis par rapport à des objectifs de santé publique et leur utilité ne saurait donc être niée.

L'article 13.1 de la loi $n^{\circ} 92.3$ sur l'eau, prévoyait l'obligation législative de leur mise en place avant le 4 janvier 1997 pour les captages existants ne bénéficiant pas d'une protection naturelle efficace mais celle-ci n'a pas été respectée. Parmi les principales raisons avancées pour justifier ce retard figurent la lourdeur et la complexité de la procédure mais, si l'on ne peut nier cette réalité, il convient également de s'interroger sur le bien-fondé de certaines servitudes lourdes proposées qui visent non pas à la stricte protection du ou des points de prélèvement d'eau mais à celle de la nappe exploitée.

Il convient donc de rappeler que, dans l'esprit du législateur, la création de périmètres de protection a pour objectif de permettre une meilleure maîtrise des risques de pollution dans la zone influencée par les prélèvements opérés et qu'ils ne peuvent apporter, en dehors de cas très particuliers (bassins d'alimentation peu étendus par exemple), une solution adaptée au problème des pollutions de type diffus (produits azotés, pesticides), celle-ci étant notamment du ressort de la réglementation générale et de l'amélioration des pratiques agricoles.

L'application, à bon escient, de la législation et de la réglementation relatives à la protection des captages d'eau destinés à l'alimentation humaine devrait permettre de surmonter les difficultés de procédure rencontrées et de rendre effective l'instauration des périmètres de protection. 